\title{
Identifying Blood Transcriptome Biomarkers of Alzheimer's Disease Using Transgenic Mice
}

\author{
Shinichiro Ochi ${ }^{1}$ - Jun-ichi Iga ${ }^{1}$ (1) $\cdot$ Yu Funahashi ${ }^{1} \cdot$ Yuta Yoshino $^{1} \cdot$ Kiyohiro Yamazaki $^{1} \cdot$ Hiroshi Kumon $^{1}$. \\ Hiroaki Mori ${ }^{1}$ - Yuki Ozaki ${ }^{1}$. Takaaki Mori ${ }^{1}$. Shu-ichi Ueno ${ }^{1}$
}

Received: 4 May 2020 / Accepted: 7 August 2020 / Published online: 20 August 2020

(C) The Author(s) 2020

\begin{abstract}
The testing of pathological biomarkers of Alzheimer's disease (AD), such as amyloid beta and tau, is time-consuming, expensive, and invasive. Here, we used 3xTg-AD mice to identify and validate putative novel blood transcriptome biomarkers of $\mathrm{AD}$ that can potentially be identified in the blood of patients. mRNA was extracted from the blood and hippocampus of 3xTg-AD and control mice at different ages and used for microarray analysis. Network and functional analyses revealed that the differentially expressed genes between $\mathrm{AD}$ and control mice modulated the immune and neuroinflammation systems. Five novel gene transcripts $(C d k n 2 a$, Apobec3, Magi2, Parp3, and Cass4) showed significant increases with age, and their expression in the blood was collated with that in the hippocampus only in AD mice. We further assessed previously identified candidate biomarker genes. The expression of Trem 1 and Trem2 in both the blood and brain was significantly increased with age. Decreased Tomm 40 and increased Pink1 mRNA levels were observed in the mouse blood. The changes in the expression of Snca and Apoe mRNA in the mouse blood and brain were similar to those found in human AD blood. Our results demonstrated that the immune and neuroinflammatory system is involved in the pathophysiologies of aging and $\mathrm{AD}$ and that the blood transcriptome might be useful as a biomarker of $\mathrm{AD}$.
\end{abstract}

Keywords Blood $\cdot$ Transcriptome $\cdot$ Biomarker $\cdot$ Hippocampus $\cdot 3 x T g-A D$ mice

\section{Introduction}

Currently, the diagnosis of Alzheimer's disease (AD) is mainly based on the assessment of clinical symptoms and cognitive tests. Several tools are used for the diagnosis of AD, and these include the identification of biomarkers from brain scans and lumbar puncture procedures. The levels of amyloid beta and tau proteins in the cerebrospinal fluid and those detected by positron emission tomography are highly accurate markers for the detection of $\mathrm{AD}$ pathology in the brain. However, the assessment of these biomarkers is invasive and time- and cost-consuming [1]. Thus, researchers are aiming to develop

Shinichiro Ochi and Jun-ichi Iga contributed equally to this work.

Electronic supplementary material The online version of this article (https://doi.org/10.1007/s12035-020-02058-2) contains supplementary material, which is available to authorized users.

Jun-ichi Iga

igajunichi@hotmail.com

1 Department of Neuropsychiatry, Molecules and Function, Ehime University Graduate School of Medicine, Shitsukawa, Toon, Ehime 791-0295, Japan simple blood tests that can accurately diagnose the disease and potentially predict its prodromal stage prior to the appearance of clinical symptoms [2].

In addition to the presence of amyloid plaques in the brain parenchyma and intraneuronal neurofibrillary tangles, emerging evidence suggests the existence of additional AD pathophysiological pathways, such as innate immune responses, neuroinflammation, and vascular and cell membrane dysregulation [3-5]. Our previous studies suggest that the detection of transcriptome biomarkers related to cell stress and inflammation in the peripheral blood has significant potential as a minimally invasive and inexpensive diagnostic tool for the diagnosis and early detection of developing $\mathrm{AD}$ [6-15]. Although DNA methylation and RNA expression changes in blood might have utility as biomarkers of cognitive dysfunction and brain aging [16, 17], the major limitation of blood biomarkers of $\mathrm{AD}$ is the lack of a direct correlation with biomarkers in brain tissues. Thus, we used $\mathrm{AD}$ model mice to identify genes that exhibit similar changes in the blood and hippocampus. If these genes are implicated in the pathophysiology of $\mathrm{AD}$, they might be good candidate blood transcriptome biomarkers of $\mathrm{AD}$.

In the present study, we simultaneously collected brain and blood samples from 3xTg-AD mice to identify novel blood 
transcriptome biomarkers of $\mathrm{AD}$ and to validate our previous biomarkers.

\section{Materials and Methods}

\section{Animal Models}

Male 3xTg-AD mice ( $n=8$ each at 12 and 52 weeks of age (w.o.a.)) and age-matched male B6129SF2 WT mice $(n=8$ each at 12 and 52 w.o.a.) were bred at Ehime University from parents originally purchased from Jackson Laboratory (3xTgAD, MMRRC \#34830; B6129SF1/J, JAX \#101043). For validation analysis, additional male $3 \times \mathrm{Tg}-\mathrm{AD}$ mice and agematched male B6129SF2 WT mice ( $n=8$ each at 36 w.o.a.) were also bred under the same conditions. All the mice were housed in a specific pathogen-free facility with climatecontrolled conditions consisting of room temperature $(22 \pm$ $2{ }^{\circ} \mathrm{C}$ ), $50 \%$ humidity. and a $12-\mathrm{h}$ light/12-h dark cycle. The mice were provided with water and a standard diet (Oriental Yeast Co., Ltd.) at libitum. At 12, 36, or 52 w.o.a., the mice were anesthetized and killed by decapitation, and 12, 36, and 52 weeks were selected as representative young, middle, and old ages based on previous studies that examined the course of pathological and behavioral changes in 3xTg AD mice [18, 19]. The hippocampi were removed and immediately stored, and blood was collected using RNeasy Protect Animal Blood Tubes (QIAGEN, \#73224) and stored at $-80{ }^{\circ} \mathrm{C}$ until RNA processing. The sample size ( $n=8$ each) was the minimum number necessary and allowed by the ethical committee. Most of the $\mathrm{AD}$ and control mice did not die before reaching 52 w.o.a. The animal experiments were approved by the Animal Experiment Committee of Ehime University (\#28-25) and were performed in accordance with the Guidelines for Animal Experiments at Ehime University.

\section{Microarray Analysis}

Total RNA was extracted from frozen hippocampi using the RNeasy Mini Kit (QIAGEN, \#74104) and from blood using the RNeasy Protect Animal Blood Kit (QIAGEN, \#73224) according to the manufacturer's instructions. The RNA concentrations were determined using a NanoDrop1000 instrument (Thermo Fisher Scientific), and its quality was assessed by determining the RNA integrity number using an Agilent 2100 Bioanalyzer (Agilent). All the samples used for microarray analysis met the following conditions: A260/A280 $\geq 1.8$, $\mathrm{A} 260 / \mathrm{A} 230 \geq 2.0$, and RNA integrity number $\geq 7.0$. Using 50 ng of RNA, amplified and biotinylated sense-strand DNA targets were generated using the Low Input Quick Amp Labeling Kit (Agilent). The hybridization, washing, and scanning steps were conducted using the Gene Expression Hybridization Kit (Agilent), Gene Expression Wash Pack
(Agilent, \#5188-5327), and Agilent Microarray Scanner (G2505C) with SurePrint G3 Mouse 8x60K version 2.0. The 75 th percentile shift normalization and baseline transformation (baseline to median of all the samples (all the blood samples or all the hippocampal samples)) were performed using Agilent GeneSpring GX version 14.9. To determine the independent and combined effects of the AD mouse model and aging, comparisons among groups (AD12 Hip (AD mouse hippocampus at 12 w.o.a.), AD52 Hip (AD mouse hippocampus at 52 w.o.a), C12 Hip (control mouse hippocampus at 12 w.o.a), and C52 Hip (control mouse hippocampus at 52 w.o.a.)) or AD12 bld )(AD mouse blood at 12 w.o.a), AD52 bld (AD mouse blood at 52 w.o.a.), C12 bld (control mouse blood at 12 w.o.a), and C52 bld (control mouse blood at 52 w.o.a)) were performed with two-way analysis of variance (ANOVA) followed by Tukey's honestly significant difference (HSD) test (two-way ANOVA: $P<0.1$, fold change $\geq 1.5$ or $\leq-1.5$, Tukey's HSD: $P<0.05$ ). The network and functional analyses were performed using IPA (QIAGEN) (https://www.qiagenbioinformatics.com/ products/ingenuity-pathway-analysis) [20]. All microarray data were deposited in the GEO database (accession number GSE144459).

\section{PCR}

For the validation of microarray data, the expression levels of various mRNAs were measured by quantitative reverse transcription-PCR (qPCR) using the StepOnePlus Real-Time PCR System (Applied Biosystems). The TaqMan probes used in this study were Mm00494449_m1 for mouse Cdkn2a and Mm99999915_g1 for mouse Gapdh (Applied Biosystems). RT-PCR was conducted using the TaqMan gene expression master mix with a final volume of $10 \mu \mathrm{L}$. The expression levels were measured in duplicate using the $\Delta \Delta \mathrm{Ct}$ method. We included the same sample in all the plates as a calibrator to adjust for differences among the plates.

\section{Validation Analyses Using Public Functional Genomics Data}

To validate biomarkers in postmortem human brain and other models of Alzheimer disease (APP PS1 model mouse), we used the expression data of Gene Expression Omnibus database (GSE48350) [21] and (GSE111737) [22], respectively. From human hippocampal data (GSE48350), young male controls $(N=9$, mean age $30.6 \pm 11.0)$, old male controls $(N=10,85.8 \pm 6.8)$, and male patients with Alzheimer's disease $(\mathrm{N}=9,84.1 \pm 6.9)$ were examined, which correspond to "C12 Hip vs. C52 Hip," "C12 Hip vs. AD52 Hip,” and "C52 Hip vs. AD52 Hip" in our data. From APP_PS1 mouse hippocampal data (GSE111737), wild-type male mice $(n=6$, 8 months old) and APP_PS1 male mice $(n=7,8$ months 
old) were examined, which correspond to "C52 Hip vs. AD52 Hip" in our data.

\section{Results}

\section{Differential Gene Expression Between Male 3xTg-AD Mice and Age-Matched Male B6129SF2 WT Mice}

The analysis of gene expression revealed positive correlations among the same tissues from the mice belonging to the same group, such as between the AD12 and AD52 hip samples, whereas negative correlations were found among the same tissues from mice belonging to different groups, such as between the $\mathrm{AD}$ and $\mathrm{C}$ hip samples. No correlations were consistently observed among different tissues, such as between the AD blood samples and the AD hip samples (Supplementary Figure 1).

We selected differentially expressed genes (DEGs) in AD mice that met the following criteria: fold change $(\geq 1.5$ or $\leq$ -1.5 ) and significance level (Tukey's HSD: $P<0.05$ ). This analysis showed that 128 and 251 were significantly increased and significantly decreased, respectively, only in the $\mathrm{AD}$ mouse blood. In contrast, 196 and genes were significantly increased and significantly decreased, respectively, only in the AD mouse hippocampus (Supplementary Table 1).

\section{Network and Functional Analyses}

To determine the functions of DEGs, network and functional analyses were conducted using IPA (Tables 1 and 2). The network analyses of the top canonical pathways showed that DEGs that were only found in the AD mouse blood were involved in "3-phosphoinositide degradation," "primary immunodeficiency signaling," and "D-myo-inositol-5-phosphate metabolism." The top upstream regulators included IL3, IL21 (inhibited), IL15RA, IFNA2 (inhibited), and TLN1. In the causal network, MAP3K13, TLR3, and androgen-AR were predicted to be inhibited, whereas KAT7 was predicted to be activated. The analysis of the top diseases and biological functions revealed that "cancer," "cellular development," and "hematological system development and function" showed the most substantial enrichment.

In contrast, the canonical pathway analysis revealed that the DEGs that were only found in the AD mouse hippocampus were involved in "dendritic cell maturation," "altered T cell and B cell signaling in rheumatoid arthritis," and "communication between innate and adaptive immune cells." The top upstream regulators included L2HGDH, lipopolysaccharide (activated), TBX5, FOSL1, and CR1L. In the causal network, L2HGDH, icilin, LY96 (activated), D-allose (inhibited), and CR1L showed the most substantial enrichment. The analysis of the top diseases and biological functions showed that "endocrine system disorders," "cell-to-cell signaling and interaction," and "hematological system development and function" exhibited the most substantial enrichment.

\section{Blood Transcriptome Biomarkers of AD}

To identify novel blood transcriptome biomarkers of $\mathrm{AD}$, we identified genes that showed significant changes with age and that exhibited correlations between the blood and hippocampus only in AD mice (Table 3). Five genes (Cdkn2a, Apobec 3, Magi2, Parp3, and Cass4) showed significant increases in expression with increasing age, and their expression in the blood was correlated with that in the hippocampus only in $\mathrm{AD}$ mice.

Our list of previously published blood transcriptome biomarkers of AD is shown in Table 4 [7-15]. The Trem 1 mRNA levels tended to increase with age only in the control mouse blood. The Trem 1 mRNA level showed significant increases with age in the control mouse brain and was significantly lower in the $\mathrm{AD}$ mouse brain than in the control mouse brain at 52 w.o.a. The Trem 2 mRNA level tended to increase with age in the control mouse blood and was lower in the AD mouse blood than in the control mouse blood at 52 w.o.a. The Trem 2 mRNA level significantly increased with age in the $\mathrm{AD}$ mouse brain and was significantly higher in the $\mathrm{AD}$ mouse brain than in the control mouse brain. The Mef2c mRNA level showed significant increases with age in the control mouse brain. The Tomm 40 mRNA level tended to decrease with age in the blood of both the AD and control mice. The Tomm 40 mRNA level significantly decreased with age in the $\mathrm{AD}$ mouse brain and was significantly lower in the $\mathrm{AD}$ mouse brain than in the control mouse brain at 52 w.o.a. The Pinkl mRNA level was significantly higher in the AD mouse blood than in the control mouse blood at both 12 and 52 w.o.a. The Pinkl mRNA level tended to increase with age and was usually lower in the AD mouse brain. The Apoe mRNA level significantly increased with age in the control mouse blood and was significantly lower in the $\mathrm{AD}$ mouse blood than in the control mouse blood at both 12 and 52 w.o.a. The Apoe mRNA level showed significant increases with age in the $\mathrm{AD}$ mouse brain and was significantly higher in the $\mathrm{AD}$ mouse brain than in the control mouse brain at 52 w.o.a. The Inpp $5 d$ mRNA level was significantly higher in the AD mouse brain than in the control mouse brain at both 12 and 52 w.o.a. The Snca mRNA level was significantly higher in the AD mouse blood than in the control mouse blood at both 12 and 52 w.o.a.

\section{Real-Time PCR Validation of DEGs in Microarray Data}

To verify the reliability of the microarray data, we selected $C d k n 2 a$ for qPCR experiments because this gene exhibited the greatest changes among the five candidate genes and is well known as an aging- and cellular senescence-associated gene $[23,24]$. This analysis aimed to not only validate the correlations between the microarray and real-time PCR data but also 
Table 1 Network and functional analyses of differentially expressed genes found only in the AD mouse blood

\begin{tabular}{|c|c|c|c|}
\hline \multicolumn{4}{|c|}{ Top canonical pathways } \\
\hline \multicolumn{2}{|c|}{ Name } & $P$ value & Overlap \\
\hline 1 & 3-phosphoinositide degradation & $1.12 \mathrm{E}-03$ & $6.2 \% 9 / 146$ \\
\hline 2 & Primary immunodeficiency signaling & $3.18 \mathrm{E}-03$ & $11.4 \% 4 / 35$ \\
\hline 3 & D-myo-inositol-5-phosphate metabolism & 4.49E-03 & $5.4 \% 8 / 147$ \\
\hline 4 & Superpathway of inositol phosphate compounds & $6.11 \mathrm{E}-03$ & $4.8 \% 9 / 188$ \\
\hline 5 & 3-Phosphoinositide biosynthesis & $6.38 \mathrm{E}-03$ & $5.1 \% 8 / 156$ \\
\hline \multicolumn{4}{|c|}{ Top upstream regulators } \\
\hline \multicolumn{2}{|c|}{ Name } & $P$ value & Predicted activation \\
\hline 1 & IL3 & $3.85 \mathrm{E}-07$ & \\
\hline 2 & IL21 & $1.38 \mathrm{E}-05$ & Inhibited \\
\hline 3 & IL15RA & $1.69 \mathrm{E}-05$ & \\
\hline 4 & IFNA2 & 4.21E-05 & Inhibited \\
\hline 5 & TLN1 & $5.55 \mathrm{E}-05$ & \\
\hline \multicolumn{4}{|c|}{ Causal network } \\
\hline \multicolumn{2}{|c|}{ Name } & $P$ value & Predicted activation \\
\hline 1 & MAP3K13 & 4.93E-07 & Inhibited \\
\hline 2 & Interferon beta-1a & $5.66 \mathrm{E}-07$ & \\
\hline 3 & TLR7 & $6.02 \mathrm{E}-07$ & Inhibited \\
\hline 4 & KAT7 & $7.48 \mathrm{E}-07$ & Activated \\
\hline 5 & Androgen-AR & $2.10 \mathrm{E}-06$ & Inhibited \\
\hline \multicolumn{4}{|c|}{ Top diseases and bio functions } \\
\hline \multicolumn{4}{|c|}{ Diseases and disorders } \\
\hline \multicolumn{2}{|c|}{ Name } & $P$ value range & Number of molecules \\
\hline 1 & Cancer & $9.16 \mathrm{E}-03-5.36 \mathrm{E}-06$ & 287 \\
\hline 2 & Hematological disease & 8.80E-03-5.36E-06 & 106 \\
\hline 3 & Immunological disease & 8.80E-03-5.36E-06 & 131 \\
\hline 4 & Organismal injury and abnormalities & $9.27 \mathrm{E}-03-5.36 \mathrm{E}-06$ & 291 \\
\hline 5 & Inflammatory response & $6.92 \mathrm{E}-03-2.02 \mathrm{E}-05$ & 46 \\
\hline \multicolumn{4}{|c|}{ Molecular and cellular functions } \\
\hline \multicolumn{2}{|c|}{ Name } & $P$ value range & Number of molecules \\
\hline 1 & Cellular development & $9.27 \mathrm{E}-03-5.57 \mathrm{E}-07$ & 111 \\
\hline 2 & Cellular growth and proliferation & $9.27 \mathrm{E}-03-5.57 \mathrm{E}-07$ & 113 \\
\hline 3 & Cell death and survival & $9.18 \mathrm{E}-03-4.80 \mathrm{E}-06$ & 117 \\
\hline 4 & Cell cycle & $9.18 \mathrm{E}-03-4.80 \mathrm{E}-06$ & 63 \\
\hline 5 & Cell-to-cell signaling and interaction & $9.27 \mathrm{E}-03-2.02 \mathrm{E}-05$ & 59 \\
\hline \multicolumn{4}{|c|}{ Physiological system development and function } \\
\hline \multicolumn{2}{|c|}{ Name } & $P$ value range & Number of molecules \\
\hline 1 & Hematological system development and function & $9.27 \mathrm{E}-03-4.13 \mathrm{E}-08$ & 84 \\
\hline 2 & Hematopoiesis & $9.27 \mathrm{E}-03-4.13 \mathrm{E}-08$ & 54 \\
\hline 3 & Tissue development & $9.27 \mathrm{E}-03-5.57 \mathrm{E}-07$ & 75 \\
\hline 4 & Embryonic development & $9.27 \mathrm{E}-03-6.04 \mathrm{E}-06$ & 57 \\
\hline 5 & Lymphoid tissue structure and development & 9.27E-03-6.04E-06 & 60 \\
\hline
\end{tabular}

confirm the detection of well-known changes. The $C d k n 2 a$ mRNA level in the blood and hippocampus significantly increased with age only in the AD mice (Fig. 1). The data $(n=8$ each group) were assessed by one-way ANOVA with Dunnett's multiple comparisons test. The significance was set to $P<0.05$. The results further validated the results of the microarray data.

\section{Validation of DEGs in the Human Postmortem Hippocampus and APP_PS1 Mouse Hippocampus}

A substantial number of candidate genes in the human postmortem hippocampus (Supplementary Figure 2) and APP_PS1 hippocampus (Supplementary Figure 3) showed similar changes to our microarray data. In particular, several 
Table 2 Network and functional analyses of differentially expressed genes found only in the $\mathrm{AD}$ mouse hippocampus

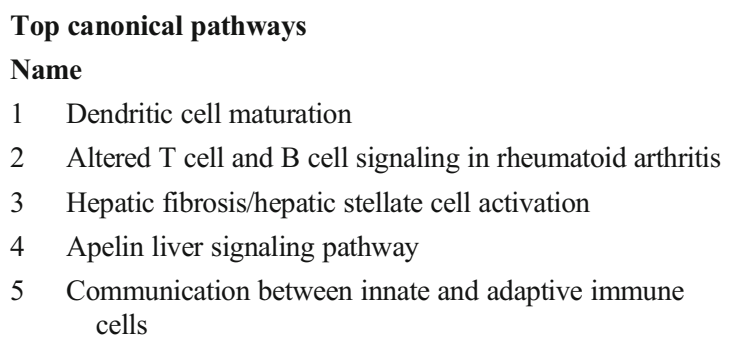

Top upstream regulators

Name

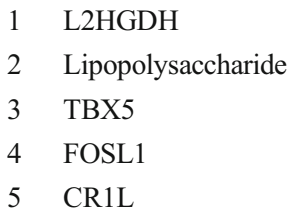

\section{Causal network}

Name

$\begin{array}{ll}1 & \text { L2HGDH } \\ 2 & \text { Icilin } \\ 3 & \text { LY96 } \\ 4 & \text { D-allose } \\ 5 & \text { CR1L }\end{array}$

Top diseases and bio functions

Diseases and disorders

\begin{tabular}{|c|c|c|c|}
\hline \multicolumn{2}{|c|}{ Name } & \multirow{2}{*}{$\begin{array}{l}\boldsymbol{P} \text { value range } \\
2.97 \mathrm{E}-03-1.71 \mathrm{E}-08\end{array}$} & \multirow{2}{*}{$\begin{array}{l}\begin{array}{l}\text { Number of } \\
\text { molecules }\end{array} \\
77\end{array}$} \\
\hline 1 & Endocrine system disorders & & \\
\hline 2 & Gastrointestinal disease & 3.09E-03-1.71E-08 & 105 \\
\hline 3 & Metabolic disease & $1.44 \mathrm{E}-03-1.71 \mathrm{E}-08$ & 51 \\
\hline 4 & Organismal injury and abnormalities & 3.23E-03-1.71E-08 & 163 \\
\hline 5 & Immunological disease & $2.97 \mathrm{E}-03-6.72 \mathrm{E}-08$ & 72 \\
\hline \multicolumn{4}{|c|}{ Molecular and cellular functions } \\
\hline \multicolumn{2}{|c|}{ Name } & $P$ value range & $\begin{array}{c}\text { Number of } \\
\text { molecules }\end{array}$ \\
\hline 1 & Cell-to-cell signaling and interaction & 2.97E-03-3.13E-09 & 54 \\
\hline 2 & Cellular movement & 3.17E-03-3.13E-09 & 62 \\
\hline 3 & Cell death and survival & 3.34E-03-8.74E-08 & 36 \\
\hline 4 & Cellular development & $2.98 \mathrm{E}-03-2.93 \mathrm{E}-06$ & 53 \\
\hline 5 & Cellular growth and proliferation & $2.98 \mathrm{E}-03-2.93 \mathrm{E}-06$ & 49 \\
\hline \multicolumn{4}{|c|}{ Physiological system development and function } \\
\hline \multicolumn{2}{|c|}{ Name } & $P$ value range & $\begin{array}{c}\text { Number of } \\
\text { molecules }\end{array}$ \\
\hline 1 & Hematological system development and function & 3.09E-03-3.13E-09 & 57 \\
\hline 2 & Immune cell trafficking & 3.09E-03-3.13E-09 & 42 \\
\hline 3 & Lymphoid tissue structure and development & $2.97 \mathrm{E}-03-2.93 \mathrm{E}-06$ & 48 \\
\hline 4 & Cell-mediated immune response & $2.49 \mathrm{E}-03-1.11 \mathrm{E}-05$ & 28 \\
\hline 5 & Connective tissue development and function & $2.98 \mathrm{E}-03-1.66 \mathrm{E}-05$ & 26 \\
\hline
\end{tabular}

$\begin{array}{ll}P \text { value } & \text { Overlap } \\ 1.63 \mathrm{E}-05 & 6.6 \% 10 / 151 \\ 4.56 \mathrm{E}-05 & 9.0 \% 7 / 78 \\ 6.99 \mathrm{E}-05 & 5.6 \% 10 / 179 \\ 2.59 \mathrm{E}-04 & 15.4 \% 4 / 26 \\ 1.25 \mathrm{E}-03 & 7.6 \% 5 / 66\end{array}$

$P$ value

3.17E-10

9.44E-08

3.08E-07

$5.73 \mathrm{E}-07$

9.23E-07

$P$ value

Predicted activation

3.17E-10

4.74E-07

4.79E-07

7.21E-07

9.23E-07

Activated

Inhibited

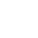




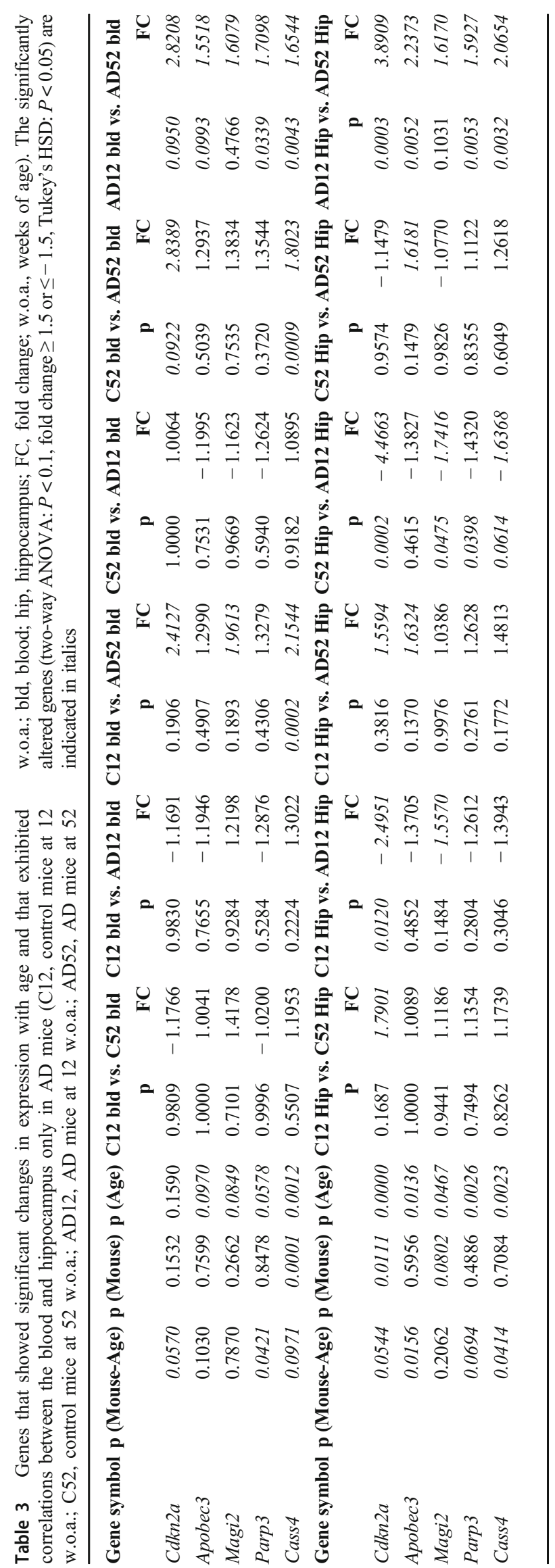




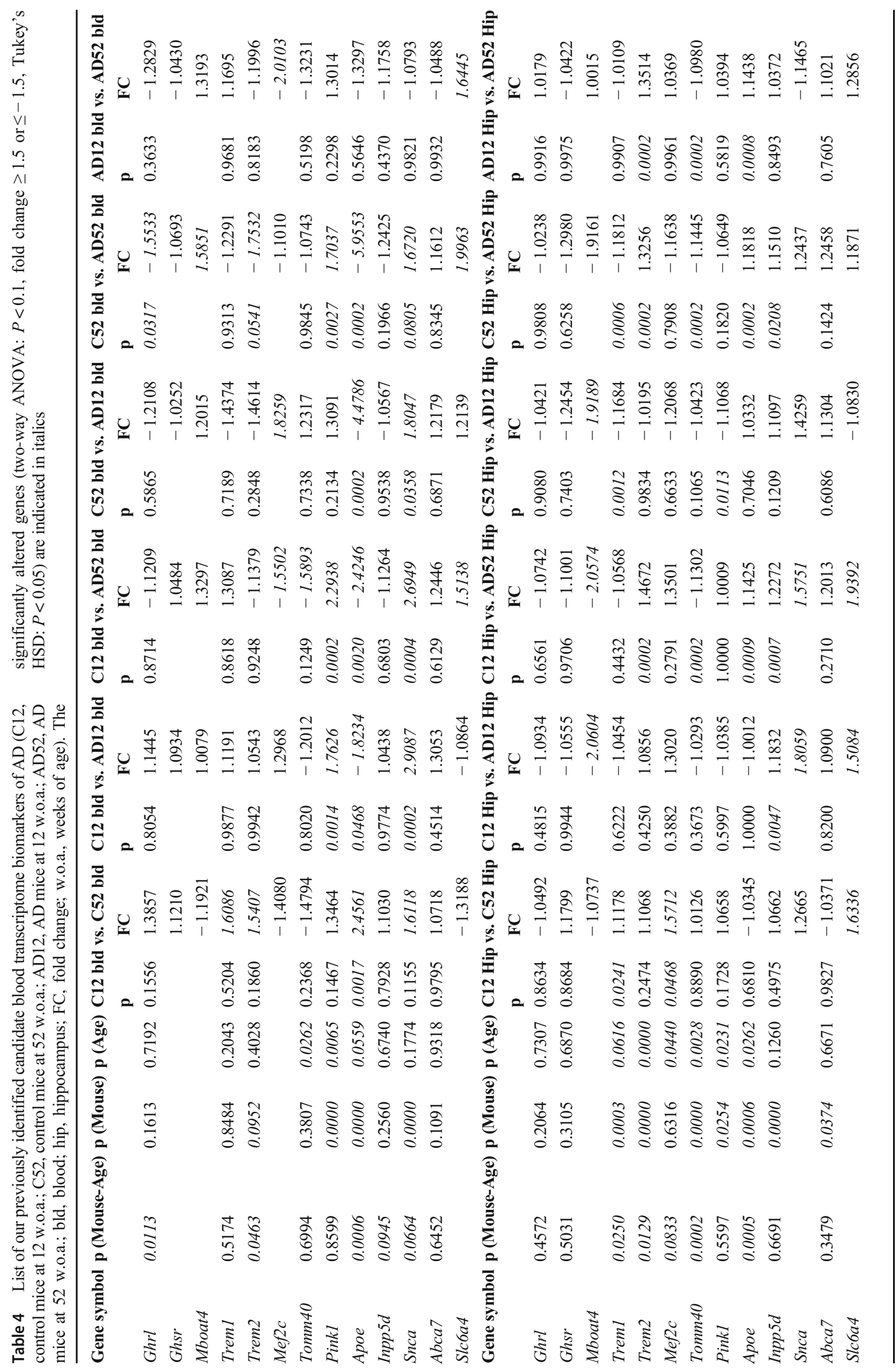


Hippocampus

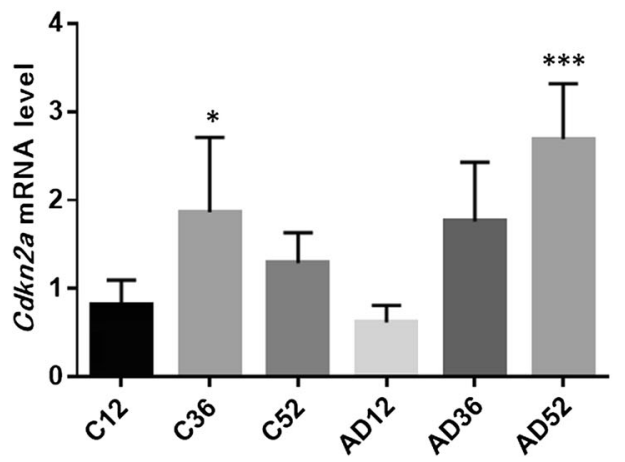

Fig. 1 Validation of $C d k n 2 a$ by qPCR. The $C d k n 2 a$ mRNA level in both the blood and hippocampus showed significant increases with age only in $\mathrm{AD}$ mice. The data $(n=8)$ are shown as the means \pm SEMs. ${ }^{*}, * *$, and *** indicate $P<0.05,0.01$, and 0.001 , respectively. * vs. the expression in $\mathrm{C} 12$ (control mice at 12 w.o.a.), as determined by one-way ANOVA

\section{Discriminant Analyses}

Discriminant analysis of the AD52 bld and C52 bld with the variables Cdkn2a, Apobec3, Magi2, Parp3, Cass4, Trem1, Trem2, Tomm40, Pink1, Apoe, Inpp5d, and Snca revealed that the combination of 3 genes (Apoe, Cass4, Cdkn2a) resulted in the best prediction (Wilks lambda $=0.170, P<0.001$ ). The discrimination score $(D)$ was calculated for each sample as follows:

$$
D=-0.697 \times \text { Apoe }+0.635 \times \text { Cass } 4+0.518 \times \mathrm{Cdkn} 2 \mathrm{a}-
$$
0.57749

The analysis showed a sensitivity and specificity of $100.0 \%$ and $100.0 \%$, respectively (Supplementary Figure 4 A).

Discriminant analysis of the AD52 Hip and C52 Hip was also performed with the same gene combination (Wilks lamb$\mathrm{da}=0.190, P<0.001)$. The discrimination score was calculated for each sample as follows:

$$
D=0.735 \times \text { Apoe }-0.113 \times \text { Cdkn2a }+0.091 \times \text { Cass } 4-
$$
0.742122

The analysis established a sensitivity and specificity of $100.0 \%$ and $100.0 \%$, respectively (Supplementary Figure 4 B).

To validate biomarkers in the blood of patients with Alzheimer's disease, we used the expression data from the Gene Expression Omnibus database (GSE97760). [25] Discriminant analysis of the AD patients $(N=9)$ and healthy subjects $(N=10)$ was conducted with the same gene combination (APOE, CASS4, CDKN2A) (Wilks lambda $=0.577$, $P=0.036$ ). The discrimination score was calculated for each sample as follows:

$D=-0.742 \times$ APOE $\times 0.696 \times \mathrm{CDKN} 2 \mathrm{~A}-0.473 \times$

CASS $4+860.715$

The analysis demonstrated a sensitivity and specificity of $77.8 \%$ and $80.0 \%$, respectively (Supplementary Figure $4 \mathrm{C}$ ).
Blood

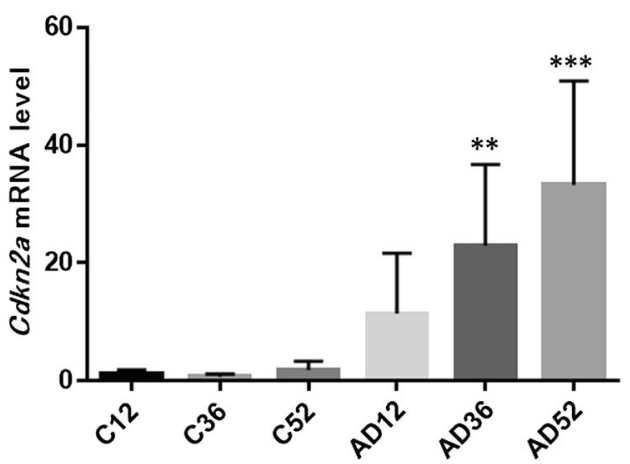

with Dunnett's multiple comparisons test. C12, control mice at 12 w.o.a.; C36, control mice at 36 w.o.a; $\mathrm{C} 52$, control mice at 52 w.o.a.; $\mathrm{AD} 12, \mathrm{AD}$ mice at 12 w.o.a.; $\mathrm{AD} 36, \mathrm{AD}$ mice at 36 w.o.a.; $\mathrm{AD} 52, \mathrm{AD}$ mice at 52 w.o.a

\section{Discussion}

The main purposes of the study were to identify novel blood transcriptome biomarkers of $\mathrm{AD}$ and to validate our previous biomarkers. Transcriptome biomarkers that exhibit significant changes in both the blood and hippocampus might have potential as minimally invasive and inexpensive markers for the diagnosis and early detection of developing AD. The lack of consistent correlations among different tissues indicates the difficulty in identifying blood biomarkers that exhibit positive correlations between the blood and hippocampus only in $\mathrm{AD}$ mice (Supplementary Figure 1). Because several genes (APOBEC3, PARP3, TREM2, TOMM40, APOE, and INPP5D) changed significantly in the same direction in multiple datasets from both human $\mathrm{AD}$ samples and mouse $\mathrm{AD}$ models, these genes may be good candidates for blood biomarkers for AD (Supplementary Figure 2 and Supplementary Figure 3). According to discriminant analyses, the combination of 3 genes (Apoe, Cass4, Cdkn2a) was the most useful to discriminate not only the blood and the hippocampus of $\mathrm{AD}$ mice but also the blood of AD patients (Supplementary Figure 4).

Interestingly, "immunological disease," "organismal injury and abnormalities," "cell-to-cell signaling and interaction," "cell death and survival," "cellular growth and proliferation," and "hematological system development and function" were the most common diseases and biofunctions found in the comparison between the blood and hippocampus. Furthermore, the DEGs that were uniquely found in the AD mouse hippocampus were involved in "altered $\mathrm{T}$ cell and B cell signaling in rheumatoid arthritis," "communication between innate and adaptive immune cells," and "immune cell trafficking." Consistently, the proinflammatory activity of microglia is related to behavioral alterations in $\mathrm{AD}$ patients and experimental models of the disease [26]. DEGs that were only found in the AD mouse blood were involved in "primary immunodeficiency signaling," "inflammatory response," and "lymphoid tissue 
structure and development." These results provide further evidence showing the important roles of the immune system in both the brain and blood in the pathogenesis of $\mathrm{AD}[27,28]$.

The expression of five gene transcripts (Cdkn2a,Apobec3, Magi2, Parp3, and Cass4) significantly increased with age, and their expression in the blood was correlated to that in the hippocampus only in AD mice (Table 3). CDKN2A is a wellknown molecular player in cellular senescence, cell proliferation, survival, adhesion, and apoptosis [23, 24]. An increased level of $C d k n 2 a$ is present in both brain and blood cells from APP/PS1 mice [29]. Interestingly, linkage and association studies have linked the CDKN2A locus (9p21.3) to lateonset $\mathrm{AD}$ families [30]. Human APOBEC3 plays important roles in intracellular defense against viral infection and cancer development by generating DNA mutations [31]. However, no previous studies have found associations between APOBEC 3 and AD. MAGI2 regulates apoptosis, cytoskeletal reorganization, and glomerular development and is broadly expressed in the brain, thyroid, and 16 other tissues, including blood [32]. MAGI2 is a candidate gene associated with multiple phenotypes, as demonstrated by Alzheimer's Disease Neuroimaging Initiative genetic studies [33]. The protein encoded by PARP3 belongs to the PARP family, and the members of this family modify nuclear proteins via polyADP-ribosylation, which is required for DNA repair, the regulation of apoptosis, and the maintenance of genomic stability [34]. Although PARP3 is a target in cancer therapy [35], no previous study has revealed an association with AD. CASS4 is a well-known candidate gene of $\mathrm{AD}$ [36] and has recently been studied in the context of immune system function and the pathogenesis of developmental and autoimmune disorders, including Crohn's disease, cancer, and other diseases [37].

Our previously identified candidate genes also showed significant changes in both the blood and brain (Table 4). The expression of Trem 1 and Trem 2 increased in the control mouse blood with age. Consistent with our previous studies, which showed higher TREM1 and TREM2 mRNA levels in the human AD blood [8-10], the levels of these mRNAs in the control mouse blood tended to increase with age. These results suggest that higher TREM1 and TREM2 mRNA levels in human AD blood might reflect abnormal aging rather than pathophysiological changes in AD. However, the identification of significant changes in the Trem 1 and Trem 2 mRNA levels in the mouse brain indicated their important roles in the development of AD. Consistent with our previous study [7], decreased Tomm 40 and increased Pink $1 \mathrm{mRNA}$ levels in mouse blood were observed in the current study. Because both TOMM40 and PINK1 play important roles in mitochondrial function, these results provide further evidence of mitochondrial dysfunction in $\operatorname{AD}[38,39]$. The Apoe mRNA level significantly increased with age in only the control mouse blood and was significantly lower in the AD mouse blood than in the control mouse blood. Although our previous study revealed that the APOE mRNA level was not changed in human AD blood [7], a recent study showed that the $A P O E$ mRNA level is significantly increased in human $\mathrm{AD}$ blood [40]. Consistent with our previous studies [41, 42], the Snca mRNA levels significantly increased with age in the control mouse blood and were significantly higher in the $\mathrm{AD}$ mouse blood than in the control mouse blood. These results indicate that the $A P O E$ and $S N C A$ mRNA levels in human blood might be candidate markers for aging and $\mathrm{AD}$.

This study has several limitations. First, our results should be validated using liquid biopsies of $\mathrm{AD}$ patients obtained from a certificated biobank. Second, because male 3xTg AD mice exhibit progressive behavioral and pathological changes at 36 and 52 w.o.a. $[14,15]$, we did not check these changes prior to tissue collection. However, the investigator who donated the $3 \times \mathrm{Tg} \mathrm{AD}$ mice communicated that in contrast to the initial observations, male transgenic mice might not exhibit the originally described phenotypic traits. Thus, we should have checked their symptoms and should also examine whether female mice also exhibit the same changes. The positive correlations in the expression of all tested genes found among the subgroups from the same group of mice suggest that the male 3xTg AD mice in this study did not lose their pathological phenotype (Supplementary Figure 1). Third, although we identified five genes that showed significant increases in expression with age and showed correlations in expression between the blood and hippocampus only in $\mathrm{AD}$ mice, we only confirmed the changes in the expression of one gene (Cdkn2a) due to sample shortage. The changes in the other four genes should be confirmed using both $\mathrm{AD}$ female mouse samples and human $\mathrm{AD}$ blood samples in future studies. Further analyses of the microarray data using complex algorithms, such as machine learning and deep learning, will be conducted in the future.

\section{Conclusion}

The results from our comprehensive analysis using brain and blood samples from transgenic mice provide insights into putative transcriptomic biomarkers of $\mathrm{AD}$. The results suggest that the expression of immune-associated genes exhibits changes in not only the blood but also the hippocampus. Because five genes (Cdkn2a, Apobec3, Magi2, Parp3, and Cass4) were detected as novel candidate transcriptomic biomarkers of $\mathrm{AD}$, these results need to be validated through further studies using human blood and brain samples. The changes in the expression of some of our candidate genes, such as Trem2, Tomm40, and Snca, in the mouse blood and brain were similar to those in the human $\mathrm{AD}$ blood. The combination of 3 genes (Apoe, Cass4, Cdkn2a) may be the most useful $\mathrm{AD}$ biomarker in the blood. This study provided further evidence showing that blood transcriptome markers have potential use as biomarkers of $\mathrm{AD}$. 
Acknowledgments We thank Ms. Chiemi Onishi for the technical assistance. This study was supported by the Division of Laboratory Animal Research and Division of Analytical Bio-Medicine at the Advanced Research Support Center (ADRES), Ehime University.

Author Contributions Conceptualization, S.O. and J.I.; methodology, J.I.; software, Y.Y. and Y.F.; validation, S.O. and J.I.; formal analysis, S.O., J.I. and Y.Y.; investigation, K.Y. and Y.O.; resources, H.K., H.M. and T.M.; writing - original draft preparation, S.O. and J.I.; writingreview and editing, J.I. and S.U.; supervision, S.U.; funding acquisition, S.O., Y.Y, J.I. and S.U. All the authors contributed to and approved the final manuscript.

Funding Information This work was supported by a Health and Labor Science Research Grant from the Japanese Ministry of Health, Labor and Welfare and a Grant-in-Aid for Scientific Research from the Japanese Ministry of Education, Culture, Sports, Science and Technology (JSPS KAKENHI Grant Numbers 17K16381, 18K07564, 18K15518, and 18H02752).

Data Availability All microarray data were deposited in the GEO database (accession number GSE144459).

\section{Compliance with Ethical Standards}

Conflict of Interest The authors declare that they have no conflict of interest.

Ethics Approval Animal experiments were approved by the Animal Experiment Committee of Ehime University (\#28-25) and were performed in accordance with the Guidelines for Animal Experiments at Ehime University.

\section{Consent for Participate Not applicable.}

Consent for Publication Not applicable.

Code Availability Not applicable.

Open Access This article is licensed under a Creative Commons Attribution 4.0 International License, which permits use, sharing, adaptation, distribution and reproduction in any medium or format, as long as you give appropriate credit to the original author(s) and the source, provide a link to the Creative Commons licence, and indicate if changes were made. The images or other third party material in this article are included in the article's Creative Commons licence, unless indicated otherwise in a credit line to the material. If material is not included in the article's Creative Commons licence and your intended use is not permitted by statutory regulation or exceeds the permitted use, you will need to obtain permission directly from the copyright holder. To view a copy of this licence, visit http://creativecommons.org/licenses/by/4.0/.

\section{References}

1. Bradfield NI, Ellis KA, Savage G, Maruff P, Burnham S, Darby D, Lautenschlager NT, Martins RN et al (2018) Baseline amnestic severity predicts progression from amnestic mild cognitive impairment to Alzheimer disease dementia at 3 years. Alzheimer Dis Assoc Disord 32(3):190-196. https://doi.org/10.1097/WAD. 0000000000000252
2. Hampel H, O'Bryant SE, Molinuevo JL, Zetterberg H, Masters CL, Lista S, Kiddle SJ, Batrla R et al (2018) Blood-based biomarkers for Alzheimer disease: mapping the road to the clinic. Nat Rev Neurol 14(11):639-652. https://doi.org/10.1038/s41582-018-0079-7

3. Heneka MT, Carson MJ, El Khoury J, Landreth GE, Brosseron F, Feinstein DL, Jacobs AH, Wyss-Coray T et al (2015) Neuroinflammation in Alzheimer's disease. Lancet Neurol 14(4): 388-405. https://doi.org/10.1016/S1474-4422(15)70016-5

4. Iturria-Medina Y, Sotero RC, Toussaint PJ, Mateos-Perez JM, Evans AC, Alzheimer's Disease Neuroimaging I (2016) Early role of vascular dysregulation on late-onset Alzheimer's disease based on multifactorial data-driven analysis. Nat Commun 7:11934. https://doi.org/10.1038/ncomms11934

5. Yulug B, Hanoglu L, Ozansoy M, Isik D, Kilic U, Kilic E, Schabitz WR (2018) Therapeutic role of rifampicin in Alzheimer's disease. Psychiatry Clin Neurosci 72(3):152-159. https://doi.org/10.1111/ pcn. 12637

6. Delvaux E, Mastroeni D, Nolz J, Chow N, Sabbagh M, Caselli RJ, Reiman EM, Marshall FJ et al (2017) Multivariate analyses of peripheral blood leukocyte transcripts distinguish Alzheimer's, Parkinson's, control, and those at risk for developing Alzheimer's. Neurobiol Aging 58:225-237. https://doi.org/10.1016/j. neurobiolaging.2017.05.012

7. Mise A, Yoshino Y, Yamazaki K, Ozaki Y, Sao T, Yoshida T, Mori $\mathrm{T}$, Mori $\mathrm{Y}$ et al (2017) TOMM40 and APOE gene expression and cognitive decline in Japanese Alzheimer's disease subjects. J Alzheimers Dis 60(3):1107-1117. https://doi.org/10.3233/JAD170361

8. Mori Y, Yoshino Y, Ochi S, Yamazaki K, Kawabe K, Abe M, Kitano T, Ozaki Y et al (2015) TREM2 mRNA expression in leukocytes is increased in Alzheimer's disease and schizophrenia. PLoS One 10(9):e0136835. https://doi.org/10.1371/journal.pone. 0136835

9. Ozaki Y, Yoshino Y, Yamazaki K, Sao T, Mori Y, Ochi S, Yoshida T, Mori T et al (2017) DNA methylation changes at TREM2 intron 1 and TREM2 mRNA expression in patients with Alzheimer's disease. J Psychiatr Res 92:74-80. https://doi.org/10.1016/j. jpsychires.2017.04.003

10. Sao T, Yoshino Y, Yamazaki K, Ozaki Y, Mori Y, Ochi S, Yoshida T, Mori T et al (2018) TREM1 mRNA expression in leukocytes and cognitive function in Japanese patients with Alzheimer's disease. J Alzheimers Dis 64(4):1275-1284. https://doi.org/10.3233/JAD180418

11. Sao T, Yoshino Y, Yamazaki K, Ozaki Y, Mori Y, Ochi S, Yoshida $\mathrm{T}$, Mori T et al (2018) MEF2C mRNA expression and cognitive function in Japanese patients with Alzheimer's disease. Psychiatry Clin Neurosci 72(3):160-167. https://doi.org/10.1111/pcn.12618

12. Yamazaki K, Yoshino Y, Mori T, Okita M, Yoshida T, Mori Y, Ozaki Y, Sao T et al (2016) Association study and meta-analysis of polymorphisms, methylation profiles, and peripheral mRNA expression of the serotonin transporter gene in patients with Alzheimer's disease. Dement Geriatr Cogn Disord 41(5-6):334 347. https://doi.org/10.1159/000447324

13. Yamazaki K, Yoshino Y, Mori T, Yoshida T, Ozaki Y, Sao T, Mori Y, Ochi S et al (2017) Gene expression and methylation analysis of ABCA7 in patients with Alzheimer's disease. J Alzheimers Dis 57(1):171-181. https://doi.org/10.3233/JAD-161195

14. Yoshino Y, Funahashi Y, Nakata S, Ozaki Y, Yamazaki K, Yoshida T, Mori T, Mori Y et al (2018) Ghrelin cascade changes in the peripheral blood of Japanese patients with Alzheimer's disease. J Psychiatr Res 107:79-85. https://doi.org/10.1016/j. jpsychires.2018.10.011

15. Yoshino Y, Yamazaki K, Ozaki Y, Sao T, Yoshida T, Mori T, Mori $\mathrm{Y}$, Ochi S et al (2017) INPP5D mRNA expression and cognitive decline in Japanese Alzheimer's disease subjects. J Alzheimers Dis 58(3):687-694. https://doi.org/10.3233/JAD-161211 
16. Chouliaras L, Pishva E, Haapakoski R, Zsoldos E, Mahmood A, Filippini N, Burrage J, Mill J et al (2018) Peripheral DNA methylation, cognitive decline and brain aging: pilot findings from the Whitehall II imaging study. Epigenomics 10(5):585-595. https:// doi.org/10.2217/epi-2017-0132

17. Qi T, Wu Y, Zeng J, Zhang F, Xue A, Jiang L, Zhu Z, Kemper K et al (2018) Identifying gene targets for brain-related traits using transcriptomic and methylomic data from blood. Nat Commun 9(1):2282. https://doi.org/10.1038/s41467-018-04558-1

18. Rae EA, Brown RE (2015) The problem of genotype and sex differences in life expectancy in transgenic AD mice. Neurosci Biobehav Rev 57:238-251. https://doi.org/10.1016/j.neubiorev. 2015.09.002

19. Gimenez-Llort L, Mate I, Manassra R, Vida C, De la Fuente M (2012) Peripheral immune system and neuroimmune communication impairment in a mouse model of Alzheimer's disease. Ann N Y Acad Sci 1262:74-84. https://doi.org/10.1111/j.1749-6632.2012. 06639.x

20. Kramer A, Green J, Pollard J Jr, Tugendreich S (2014) Causal analysis approaches in ingenuity pathway analysis. Bioinformatics 30(4):523-530. https://doi.org/10.1093/bioinformatics/btt703

21. Berchtold NC, Cribbs DH, Coleman PD, Rogers J, Head E, Kim R, Beach T, Miller C et al (2008) Gene expression changes in the course of normal brain aging are sexually dimorphic. Proc Natl Acad Sci U S A 105(40):15605-15610. https://doi.org/10.1073/ pnas.0806883105

22. Fang EF, Hou Y, Palikaras K, Adriaanse BA, Kerr JS, Yang B, Lautrup S, Hasan-Olive MM et al (2019) Mitophagy inhibits amyloid-beta and tau pathology and reverses cognitive deficits in models of Alzheimer's disease. Nat Neurosci 22(3):401-412. https://doi.org/10.1038/s41593-018-0332-9

23. Misawa T, Tanaka Y, Okada R, Takahashi A (2020) Biology of extracellular vesicles secreted from senescent cells as senescenceassociated secretory phenotype factors. Geriatr Gerontol Int 20(6): 539-546. https://doi.org/10.1111/ggi.13928

24. LaPak KM, Burd CE (2014) The molecular balancing act of p16(INK4a) in cancer and aging. Mol Cancer Res 12(2):167-183. https://doi.org/10.1158/1541-7786.MCR-13-0350

25. Naughton BJ, Duncan FJ, Murrey DA, Meadows AS, Newsom DE, Stoicea N, White P, Scharre DW et al (2015) Blood genome-wide transcriptional profiles reflect broad molecular impairments and strong blood-brain links in Alzheimer's disease. J Alzheimers Dis 43(1):93-108. https://doi.org/10.3233/JAD-140606

26. Selles MC, Oliveira MM, Ferreira ST (2018) Brain inflammation connects cognitive and non-cognitive symptoms in Alzheimer's disease. J Alzheimers Dis 64(s1):S313-S327. https://doi.org/10. 3233/JAD-179925

27. Golde TE (2019) Harnessing immunoproteostasis to treat neurodegenerative disorders. Neuron 101(6):1003-1015. https://doi.org/10. 1016/j.neuron.2019.02.027

28. Sabatino JJ Jr, Probstel AK, Zamvil SS (2019) B cells in autoimmune and neurodegenerative central nervous system diseases. Nat Rev Neurosci 20(12):728-745. https://doi.org/10.1038/s41583019-0233-2

29. Esteras N, Bartolome F, Alquezar C, Antequera D, Munoz U, Carro E, Martin-Requero A (2012) Altered cell cycle-related gene expression in brain and lymphocytes from a transgenic mouse model of Alzheimer's disease [amyloid precursor protein/presenilin 1 (PS1)].
Eur J Neurosci 36(5):2609-2618. https://doi.org/10.1111/j.14609568.2012.08178.x

30. Zuchner S, Gilbert JR, Martin ER, Leon-Guerrero CR, Xu PT, Browning C, Bronson PG, Whitehead P et al (2008) Linkage and association study of late-onset Alzheimer disease families linked to 9p21.3. Ann Hum Genet 72(Pt 6):725-731. https://doi.org/10. 1111/j.1469-1809.2008.00474.x

31. Green AM, Weitzman MD (2019) The spectrum of APOBEC3 activity: From anti-viral agents to anti-cancer opportunities. DNA Repair (Amst) 83:102700. https://doi.org/10.1016/j.dnarep.2019. 102700

32. Empitu MA, Kadariswantiningsih IN, Aizawa M, Asanuma K (2018) MAGI-2 and scaffold proteins in glomerulopathy. Am J Physiol Ren Physiol 315(5):F1336-F1344. https://doi.org/10. 1152/ajprenal.00292.2018

33. Shen L, Thompson PM, Potkin SG, Bertram L, Farrer LA, Foroud TM, Green RC, Hu X et al (2014) Genetic analysis of quantitative phenotypes in $\mathrm{AD}$ and MCI: imaging, cognition and biomarkers. Brain Imaging Behav 8(2):183-207. https://doi.org/10.1007/ s11682-013-9262-z

34. Belousova EA, Ishchenko capital A C, Lavrik OI (2018) Dna is a new target of Parp3. Sci Rep 8(1):4176. https://doi.org/10.1038/ s41598-018-22673-3

35. Rodriguez-Vargas JM, Nguekeu-Zebaze L, Dantzer F (2019) PARP3 comes to light as a prime target in cancer therapy. Cell Cycle 18(12):1295-1301. https://doi.org/10.1080/15384101.2019. 1617454

36. Kim JH (2018) Genetics of Alzheimer's disease. Dement Neurocogn Disord 17(4):131-136. https://doi.org/10.12779/dnd. 2018.17.4.131

37. Deneka A, Korobeynikov V, Golemis EA (2015) Embryonal Fynassociated substrate (EFS) and CASS4: the lesser-known CAS protein family members. Gene 570(1):25-35. https://doi.org/10.1016/j. gene.2015.06.062

38. Guo L, Tian J, Du H (2017) Mitochondrial dysfunction and synaptic transmission failure in Alzheimer's disease. J Alzheimers Dis 57(4):1071-1086. https://doi.org/10.3233/JAD-160702

39. Hou Y, Dan X, Babbar M, Wei Y, Hasselbalch SG, Croteau DL, Bohr VA (2019) Ageing as a risk factor for neurodegenerative disease. Nat Rev Neurol 15(10):565-581. https://doi.org/10.1038/ s41582-019-0244-7

40. Chen YC, Chiu YJ, Lin CH, Hsu WC, Wu JL, Huang CH, Lin CW, Yao CF et al (2019) Indole compound NC009-1 augments APOE and TRKA in Alzheimer's disease cell and mouse models for neuroprotection and cognitive improvement. J Alzheimers Dis 67(2): 737-756. https://doi.org/10.3233/JAD-180643

41. Funahashi Y, Yoshino Y, Yamazaki K, Mori Y, Mori T, Ozaki Y, Sao T, Ochi S et al (2017) DNA methylation changes at SNCA intron 1 in patients with dementia with Lewy bodies. Psychiatry Clin Neurosci 71(1):28-35. https://doi.org/10.1111/pcn.12462

42. Yoshino Y, Mori T, Yoshida T, Yamazaki K, Ozaki Y, Sao T, Funahashi Y, Iga JI et al (2016) Elevated mRNA expression and low methylation of SNCA in Japanese Alzheimer's disease subjects. J Alzheimers Dis 54(4):1349-1357. https://doi.org/10.3233/ JAD- 160430

Publisher's Note Springer Nature remains neutral with regard to jurisdictional claims in published maps and institutional affiliations. 